\title{
Un regard méthodique sur le tarif et son adaptation
}

\section{Andreas Bührer}

Dr sc. nat. et med., Andreas Bührer Consulting, Weingarten-Kalthäusern

Applicable à toute la Suisse, la structure tarifaire se fonde sur le modèle tarifaire du TARMED. La valeur du point tarifaire, c'est-à-dire le prix, fait l'objet de négociations pour chaque communauté contractuelle. Dans la LAMal, les coûts globaux correspondent au produit du "volume" x le "prix». Par conséquent, ils ne peuvent pas uniquement être régulés par la structure tarifaire et par les tarifs.

Le modèle tarifaire TARMED garantit la conformité du tarif aux critères de l'économie d'entreprise.

Le modèle tarifaire se fonde sur une approche économique et sur des algorithmes conformes aux critères de l'économie d'entreprise. De manière générale, les modèles ont pour tâche de représenter la réalité de manière aussi fidèle que possible.

La structure tarifaire est le résultat de l'application du modèle tarifaire.

La structure tarifaire est le résultat de l'application du modèle tarifaire. Elle se compose de la nomenclature, de l'évaluation de la prestation pour chaque position et du nombre de points tarifaires requis pour la prestation médicale (PM) et la prestation technique (PT). Un point tarifaire représente un franc suisse.

L'actualisation du tarif est essentielle.

Une structure tarifaire doit être régulièrement révisée et actualisée, faute de quoi elle devient obsolète et ne correspond plus à la réalité. Or la structure tarifaire TARMED n'a jamais été entièrement révisée depuis son introduction il y a plus de dix ans, et la structure tarifaire actuelle (1.08.00_BR) ne tient donc pas compte de l'évolution des coûts. Par conséquent, il est fort probable qu'une nouvelle structure tarifaire, fondée sur un nouveau calcul économique, génère des points tarifaires plus élevés, ne serait-ce qu'en raison de l'évolution salariale du personnel non médical.
Sur le plan méthodique, les interventions dans la structure tarifaire revêtent un caractère arbitraire.

Sur le plan strictement méthodique, les interventions directes dans la structure tarifaire revêtent un caractère arbitraire car elles ne tiennent pas compte des critères, règles et conventions économiques qui soustendent le modèle tarifaire TARMED et qui permettent de garantir une structure tarifaire appropriée répondant aux règles applicables en économie d'entreprise.

Les valeurs du point tarifaire sont plus basses pour les cas de maladie et les cabinets.

Les valeurs de point tarifaire appliquées (dans la mesure où elles ont été rendues publiques) oscillent entre $0.75 \mathrm{CHF}$ et $1.00 \mathrm{CHF}$ et il s'avère que les mêmes prestations sont nettement moins bien rémunérées dans l'assurance-maladie que dans l'assurance-accident et dans les cabinets médicaux que dans les hôpitaux.

Les coûts globaux ne peuvent pas être régulés uniquement par la structure tarifaire et par les tarifs, même si le principe du caractère économique est respecté.

Question centrale dans le domaine de l'assurancemaladie, les coûts globaux correspondent au produit $\mathrm{du}$ "volume» $\times$ la valeur du point tarifaire $\times$ le nombre de points tarifaires, le facteur principal de ce calcul étant celui du «volume». A cet égard, il serait réducteur de considérer que l'augmentation du «volume» résulte exclusivement de facteurs économiques. Les facteurs d'influence sont très divers et comprennent par 
exemple aussi les attentes de la population, l'influence des médias et de la publicité, la qualité, la réglementation, la responsabilité globale ou la manière d'envisager des questions comme celles de la fin de vie et du décès. Or le "volume» et donc les coûts globaux peuvent difficilement être régulés par la structure tarifaire et par les tarifs même si le principe du caractère économique est respecté.

\section{Aucun budget global n'est prévu} pour le domaine ambulatoire de la LAMal.

La LAMal ne prévoit aucun budget global pour le domaine ambulatoire, y compris parmi les mesures extraordinaires visant à endiguer les coûts [1]. Aujourd'hui, un budget global ne pourrait pas être mis en place, faute d'expériences, d'approches et de concepts concrets pour savoir précisément comment employer et répartir les moyens financiers à tous les groupes de population en respectant l'éthique médicale. De plus, une réduction de la valeur du point tarifaire représenterait une solution ni viable ni appropriée pour procéder à d'éventuelles corrections en cas de dépassement du budget.

\section{La neutralité des coûts ne s'applique qu'aux changements de modèle.}

Le budget global ne doit pas être confondu avec la «neutralité des coûts lors d'un changement de modèle» [2]. Sur le plan de la méthode, on parle de neutralité des coûts lorsque le calcul pour déterminer la rémunération des prestations LAMal fournies au cours d'une période définie, par ex. une année, indique des coûts globaux identiques, que le calcul soit effectué selon l'ancien ou le nouveau modèle. Un changement de modèle neutre en termes de coûts ne dit rien de l'évolution ultérieure des coûts. En revanche, plus on intro- duit de facteurs prospectifs dans le calcul, plus on se rapproche d'un budget global caché. Par ailleurs, du point de vue de la méthode, l'ancien modèle devrait d'abord être révisé avant de passer au nouveau modèle, faute de quoi les deux modèles ne refléteraient pas la même réalité. Or comment l'ancienne structure tarifaire, qui n'est plus appropriée - faute de mise à jour -, pourrait-elle former une base appropriée pour la nouvelle structure tarifaire?

\section{L'exigence de neutralité des coûts lors de révisions tarifaires remet en question le fondement économique et le caractère approprié du tarif.}

Il ne faut pas non plus confondre neutralité des coûts lors d'un changement de modèle et révision tarifaire. Sur le plan de la méthode, aucune neutralité des coûts n'est possible lors d'une révision tarifaire, car cela nuirait à la conformité du modèle avec les critères de l'économie d'entreprise.

\section{La double neutralité des coûts a tout d'un budget global.}

Même si elle n'est pas applicable lors d'une révision tarifaire, la neutralité des coûts est souvent exigée sous une forme dite "statique». En outre, une neutralité des coûts dite «dynamique» est également exigée afin de limiter l'évolution du volume de prestations d'une année à l'autre. Or cette double neutralité des coûts a tout d'un budget global, à cela près que les autorités tarifaires n'assument pas la responsabilité de l'affectation des moyens financiers.

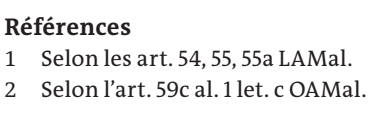

